\title{
MicroRNAs as Regulators and Biomarkers of Platelet Function and Activity in Coronary Artery Disease
} \author{
Stefan Stojkovic ${ }^{1}$ Anne Yaël Nossent ${ }^{1}$ Paul Haller $^{2}$
Johann Wojta ${ }^{1,4,5} \quad$ Kurt Huber $^{2,3}$
${ }^{1}$ University Clinic for Internal Medicine II, Department of Cardiology,
Medical University of Vienna, Vienna, Austria \\ 23rd Department of Internal Medicine, Cardiology, Intensive Care \\ Medicine and Chest Pain Unit, Wilhelminenhospital, Vienna, Austria \\ ${ }^{3}$ Medical Faculty, Sigmund Freud University, Vienna, Austria \\ ${ }^{4}$ Core Facilities, Medical University of Vienna, Vienna, Austria \\ ${ }^{5}$ Ludwig Boltzmann Cluster for Cardiovascular Research, Vienna \\ Austria
}

Thromb Haemost 2019;119:1563-1572.

\author{
Address for correspondence Stefan Stojkovic, PhD, University Clinic \\ for Internal Medicine II, Department of Cardiology, Medical University \\ of Vienna, Währinger Gürtel 18-20, A-1090 Vienna, Austria \\ (e-mail: stefan.stojkovic@meduniwien.ac.at).
}

\author{
Abstract \\ Keywords \\ - microRNA \\ - platelet function \\ - coronary artery \\ disease \\ - acute myocardial \\ infarction \\ - acute coronary \\ syndrome
}

Microribonucleic acids (miRs) are small, noncoding ribonucleic acids (RNAs), which play an important role in the regulation of platelet function and activity. Several studies proposed a mechanistic role of platelet-related miRs in the pathophysiology of coronary artery disease (CAD) and atherothrombosis. Circulating, platelet-related miRs have been proposed as diagnostic, prognostic, as well as treatment response biomarkers in CAD and acute coronary syndrome (ACS). In this review, we summarize recent studies on the role of platelet-related miRs in the regulation of platelet function and activity. Furthermore, we review the studies investigating the role of plateletrelated miRs as biomarkers in patients with CAD and ACS.

\section{Introduction}

Microribonucleic acids (miRs) are small, noncoding ribonucleic acids (RNAs) that regulate posttranscriptional gene expression by inhibiting translation or causing degradation of their target messenger RNA (mRNA). ${ }^{1}$ MiRs bind to the $3^{\prime}$ untranslated region (UTR) of their target mRNA through the so-called "seed region" on nucleotides 2 to 8 . Because this short binding site sequence can be present on many mRNAs, a single $\mathrm{miR}$ is able to target and regulate hundreds of mRNAs. ${ }^{2}$ Since miRs primarily affect mRNA transcript stability, resulting in small changes in protein levels, effects on a single miR-target might be subtle. ${ }^{1}$ However, the combined effect of miRs on multiple targets within a regulatory network could change the output of a pathway. ${ }^{1}$

received

March 11, 2019

accepted after revision

June 12, 2019

The expression of miRs is regulated in a tissue- and pathology-specific manner. MiRs are present in the human circulation in cell-free form, where they can be detected with high sensitivity and specificity using sequence-specific amplification, and thus may serve as potential biomarkers in various disease pathologies. ${ }^{3}$ Circulating miRs are stable in the circulation and resistant to endogenous ribonuclease activity. MiRs are either transported in extracellular vesicles like exosomes, microvesicles (MVs), and apoptotic bodies, or can be found in protein/lipoprotein complexes with highdensity lipoprotein (HDL), argonaute2, a catalytic component of the RNA-induced silencing complex, and nucleophosmin, a ubiquitously expressed phosphoprotein of the nucleolus. ${ }^{4-7}$ Because of their remarkable stability in circulation, several studies have proposed various miRs as

(c) 2019 Georg Thieme Verlag KG Stuttgart · New York
DOI https://doi.org/ 10.1055/s-0039-1693702. ISSN 0340-6245. 
biomarkers for early detection of acute myocardial infarction (AMI), stable coronary artery disease (CAD), in-stent restenosis, outcome in acute and chronic heart failure, and for cardiovascular risk factors such as type 2 diabetes and obesity. ${ }^{8-15}$

\section{Platelets Express Functional microRNAs}

Platelets are anucleate cells, and although it has been known that they contain functional pre-mRNA and mRNA and produce proteins, platelets were not the first place to start looking for miRs expression. However, the presence of miRs in platelets was first reported already in 2008. Merkerova et al reported differential expression of 13 common miRs over various types of peripheral blood-derived cell types, including platelets. ${ }^{16}$ Out of the selected 13 miRs, miR-223 was abundantly expressed in platelets, as well as in granulocytes and monocytes. The authors focused mainly on using differential miRs profiles to differentiate between different hematopoietic lineages. In 2009, the phenomenon of miRs expression in platelets was examined specifically. Landry et al showed that platelets express a broad array of different miRs. ${ }^{17}$ More importantly, however, the authors demonstrated that platelets did not merely inherit some miRs from their megakaryocyte progenitors, but that they have a functional miRs machinery, capable of processing miRs, as well as binding and targeting mRNAs. More recently, Rowley et al showed that megakaryocyte-specific knockout of Dicer, a key component of the miRs machinery, leads to a broad-scale reduction in platelet miRs. ${ }^{18}$ As a result, platelet protein expression was altered as well. Although it cannot be excluded that some of these changes were inherited from the knockout parent megakaryocytes, this study strongly indicates a regulatory role of miRs in platelet protein translation.

Nagalla et al performed a screen for miRs expression in platelets isolated from 19 healthy volunteers. ${ }^{19}$ They found that overall, platelets expressed 284 different miRs. The authors also looked at mRNA expression using whole genome microarray analysis and compared miRs and mRNA expression for microRNA-mRNA pairs with predicted binding of the miRs to the mRNA's 3'UTR. Expression of miRs and target mRNAs were inversely correlated. The authors selected three putative targets for three platelet miRs and showed that protein expression was also downregulated, clearly indicating normal miRs functionality in anucleate platelets. With regards to platelet functionality, the authors showed that 74 miRs were differentially expressed among platelets from individuals with high, versus individuals with low platelet aggregation in response to epinephrine. A very similar study was published by Osman and Fälker in the same year, in which they identified 281 miRs in platelets. ${ }^{20}$ Upon platelet activation with thrombin, 6 miRs, namely miR15a, miR-339, miR-365, miR-495, miR-98, and miR-361-3, showed differential expression.

To study the stability of miRs expression in platelets at different time points, Stratz et al looked at miRs expression in platelets from 5 healthy volunteers collected at 5 different time points over 10 consecutive days. ${ }^{21}$ Furthermore, at
24 hours before the last blood draw, volunteers were given a single dose of $500 \mathrm{mg}$ aspirin. Although more miRs were detected, a total of $221 \mathrm{miRs}$ was detected in the platelets of all 5 volunteers at all 5 time points. The authors found that miRs expression was remarkably stable between individuals and between the different time points. Furthermore, after 24 hours, there were no significant effects of aspirin on miRs expression in the 5 individuals included in this study. However, one could speculate that changes in miRs expression might occur later than 24 hours after aspirin intake. In 2012, the first RNA-sequencing data on miRs in platelets were published. Plé et al detected at least 492 different mature miRs in platelets, including 40 novel, previously undescribed miRs. ${ }^{22}$ Moreover, the authors demonstrated the occurrence of posttranscriptional modifications in platelet miRs, as well many iso-miRs formed by small shifts in pri- and pre-miRs cleavage site selection by Drosha and Dicer, respectively. Most of the posttranscriptional modifications found in platelets impact mostly miRs processing rates and miRs stability. But iso-miRs with nucleotide additions or deletions at the 5 'end cause alterations in the miRs' seed sequence, and thus in the miRs' set of target mRNAs. The discovery of miRs modifications and iso-miRs only adds to the complexity of translational regulation in anucleate platelets.

While Stratz et al had shown platelets to be stable over time in 5 volunteers of the same sex and age, Simon et al studied whether platelet miRs levels were altered by age and sex. $^{23}$ The authors showed that 15 platelet miRs were differentially expressed over different ages, as well as 9 miRs that were differentially expressed between men and women. The authors further showed that putative target mRNAs of these miRs were also differentially expressed, in an inverse manner. Even though these results were obtained in a cohort of individuals with Caucasian and African descent, the authors did not report differential expression between both groups. Another study, looking specifically at differences between European and African descendants, however, did report differences in platelet miRs levels and function between both races. ${ }^{24}$

\section{MicroRNA Functions within Platelets}

The above described studies mostly look at global plateletmRNA and platelet-miRNA expression, allowing for normalization to the total mRNA or miRs expression. However, when studying expression of individual mRNAs and miRs, normalization to stably expressed housekeeping genes is essential, particularly in platelets. Platelet activation leads to rapid changes in RNA (and protein) expression, via pre-mRNA splicing ${ }^{25}$ and via processing of miRs precursors (unpublished data). As platelets have no transcription, these changes are not controlled by the cellular machinery. In discussing papers on platelet functionality, we will report the chosen normalization strategies, as these could impact the interpretation of the results. However, one should keep in mind that an optimal normalization strategy does not exist (yet).

When discussing platelet functionality, one should also keep in mind that there are many ways to measure platelet 
activity. In vivo assays in mice include tail bleeding time, clot formation (both speed and size) in the carotid artery after $\mathrm{FeCl}_{3}$-induced vascular injury, counting embolisms in the microvasculature, and counting platelet-leukocyte aggregates. These in vivo tests are probably most relevant as disease models, but also nonspecific and indirect as other components in the blood and vessel wall could influence the outcomes. In vitro platelet aggregation in response to a specific agonist, as well as platelet intracellular calcium mobilization assays, are more direct ways of measuring platelet activation. But as platelets are easily activated in vitro, these tests are probably less clinically relevant. Alternatively, in vitro/ex vivo clot formation can be monitored, which is more clinically relevant. However, it becomes clear from the discrepancies between the studies by Elgheznawy et al and Leierseder et al, all in vitro platelet assays are highly sensitive to the assay conditions. ${ }^{26,27}$

- Table 1 gives overview of the key platelet miRs and their function within platelets. As described above, Edelstein et al looked at differences in platelet activation between individuals with European and African descent. ${ }^{24}$ The authors found that protease-activated receptor 4 (PAR4) activation of platelets was enhanced in African descendants, leading to increased platelet aggregation in vitro. The authors demonstrated that phosphatidylcholine transfer protein (PC-TP), among others, was responsible for the enhanced platelet activation via PAR4 in those with African descent. The authors also show that PC-TP is targeted by miR-376c, which is also differentially expressed between both races. However, a direct effect of miR-376c inhibition or overexpression on platelet activation was not shown. MiR-376c is part of a large polycistronic gene cluster encoding well over 50 different microRNA genes. This cluster is located on the long arm of chromosome 14 (14q32) within the DIO3-DLK1 locus. It has been implicated in cardiovascular disease and vascular remodeling frequently. ${ }^{28}$ Edelstein et al reported that $14 \mathrm{q} 32$ miRs were generally downregulated in individuals of African descent, potentially allowing for upregulation of their targets, including PC-TP. In this study, mRNA levels were normalized to $\beta$-actin, and miRs levels to RNU6B.

Looking at miRs function in platelets in a disease setting, Luo et al showed that hyperglycemia in type 2 diabetes reduces expression of miR-30c in platelets. ${ }^{29}$ The authors further show that plasminogen activator inhibitor-1 (PAI-1) is direct target of miR-30c and is upregulated in platelets of diabetic mice, and in human patients with type II diabetes. Lentiviral overexpression of miR-30c led to reduced arterial occlusion in a murine $\mathrm{FeCl}_{3}$-induced arterial thrombosis model in a PAI-1-dependent manner, indicating a causal relationship between miR-30c and platelet function. In this study, mRNA levels were normalized to $18 \mathrm{~S}$ ribosomal RNA (rRNA), miRNA levels to U6 small nuclear RNA.

Elgheznawy et al looked at effects of type 2 diabetes on platelet miRs in a broader sense. The authors studied the effects of calpain, a calcium-dependent protease that is activated in platelets in type 2 diabetes. ${ }^{26} \mathrm{~A}$ previous study had shown that calpain can cleave and inactivate Dicer. Indeed, both in human diabetic patients and in diabetic mice, Dicer, as well several platelet miRs were decreased. In mice, these decreases could be reversed by treatment with a calpain inhibitor. The authors focused on the function of one of the downregulated miRs, miR-223. MiR-223 deficient mice displayed enhanced in vivo platelet-neutrophil aggregate formation. Furthermore, miR-223 deficient mice displayed accelerated formation of larger clots in response to $\mathrm{FeCl}_{3}$-induced injury of the carotid artery, as well as an increased number of emboli in the microcirculation. In vitro a modest increase in platelet aggregation, accompanied by the formation of larger thrombi was observed. These results contradict an earlier study on miR-223, the most abundantly expressed platelet microRNA. In 2013, Leierseder et al published that miR-223 deficiency had absolutely no effect whatsoever on platelet function, not on activation, nor on platelet aggregation. ${ }^{27}$ However, some differences in study design between both studies may help explain these discrepancies. Where Elgheznawy et al found their most striking effects on platelet function in vivo, Leierseder et al looked only at in vitro models. Furthermore, Elgheznawy et al reported enhanced in vitro platelet aggregation in miR-223 deficient platelets in response to thrombin, but only at concentrations below $0.75 \mathrm{U} / \mathrm{mL}$. Leierseder et al reported no differences in in vitro platelet aggregation, but only tested this at a concentration of $0.75 \mathrm{U} / \mathrm{mL}$. Leierseder et al normalized miR-223 levels to U6, whereas Elgheznawy et al used $18 \mathrm{~S}$ rRNA for normalization.

The results regarding the effects of type 2 diabetes on calpain and miR-223 were confirmed in study by Fejes et al. $^{30}$ The authors show that platelet miR-223, miR-26b, miR-126, and miR-140 are downregulated in patients with type 2 diabetes in part via Dicer cleavage by calpain. The authors identify purinergic receptor P2Y12 (P2RY12) as target mRNA for miR-223 and P-selectin as target for miR-26b and miR-140. Furthermore, it was demonstrated that diabetic platelets are more active, but platelet activity was determined only by surface P-selectin expression. The expression levels of miRs were normalized to RNU43. ${ }^{30}$ A very recent study by Garcia et al, confirms the previous finding of Kaudewitz et al that miR-126-3p regulates disintegrin and metalloproteinase domain-containing protein 9 expression in these cells. ${ }^{31,32}$ Furthermore, it brings further insight on the role of miR-126 in platelets, by showing that miR-126-3p targets plexin-B2 in platelet-like structures differentiated from primary human CD34 + -derived megakaryocytes. ${ }^{31}$ Upregulation of miR$126-3 p$, as well as downregulation of plexin-B2, led to an increase in platelet reactivity, defined by increased immobilization on a fibrinogen-coated surface under flow. MiR-126-3p expression was normalized to a panel of three miRs, consisting of miR-28, miR-151, and miR-29c. ${ }^{31}$

Although platelets are difficult to work with in vitro, several other studies have investigated miRs function in platelets. Kondkar et al showed that upregulation of the gene VAMP8, which encodes the protein endobrevin, is associated with platelet hyperreactivity, measured by in vitro platelet aggregation. ${ }^{33}$ The authors demonstrated that VAMP8 is targeted by miR-96, which is abundantly expressed in platelets. A direct link between miR-96 and 
VAMP8, however, was shown in HCT116-Dicer-KO2 cells, rather than in platelets. In this study, both mRNA and miRs levels were normalized to glyceraldehyde 3-phosphate dehydrogenase (GAPDH).

Zhou et al looked at an opposite phenotype, namely at hypo-responding platelets to activation via the Fc receptor for immunoglobulin G IIA (FcrRIIA). ${ }^{34}$ The authors observed upregulation of platelet $\mathrm{T}$ cell ubiquitin ligand-2 (TULA-2). TULA-2 was shown to be targeted by miR-148a and inhibition of miR-148a led to increased platelet TULA-2 expression in FcrRIIA transgenic mice, as well as to reduced thrombus formation in vivo in response to FcrRIIA activation. Surprisingly, however, the authors do not report miR-148a expression levels at all, which could make one question whether the conclusion that miR-148a directly influences platelet function is justified.

Platelets have multiple activation routes, each with different outcomes. Miao et al looked at activation of platelets via thrombin. ${ }^{35}$ In this study, mRNA levels were normalized to GAPDH, and miRs levels were normalized to miR-130b or RNU6B. Thrombin activation of platelets in vitro led to downregulation of miR-27b and the authors demonstrated that miR-27b upregulation using microRNA mimics leads to specific thrombospondin-1 downregulation. Thrombospondin-1 has antiangiogenic properties, and indeed, lysate of platelets treated with miR-27b mimics increased Matrigeltube formation by endothelial progenitor cells in vitro. The authors demonstrated a function for miR-27b within platelets, but the ultimate effect, angiogenesis, would occur in other tissues. A similar mechanism was demonstrated by Barwari et al, regarding platelet miR-21. MiR-21 was implicated in tissue fibrosis already in $2008 .{ }^{36} \mathrm{~A}$ decade later, Barwari et al show that platelet miR-21 plays an important role in fibrosis in other tissues. ${ }^{37}$ The authors show that miR21 targets the Wiskott-Aldrich syndrome protein in platelets, which is a negative regulator of platelet transforming growth factor (TGF)- $\beta 1$ secretion. Via regulation of TGF- $\beta 1$ release, platelet miR-21 could indirectly contribute to fibrosis in other tissues. In this study, different housekeeping genes were selected based on analysis of stability.

\section{Transfer of Platelet microRNAs to Other Cells}

Platelet miRs have a direct way of contributing to homeostasis in other tissues. Namely, platelets produce vast amounts of MVs upon stimulation and these MVs contain functional miRs. Ambrose et al published very recently that the exact composition of the microRNA load of these vesicles is even determined, to some extent, by the route of platelet activation. ${ }^{38}$ Several studies have now shown that these MVs and their microRNA load are actively transferred to endothelial cells, smooth muscle cells (SMCs), and macrophages, where they target host cells' mRNAs. Gidlöf et al were the first to show uptake of platelet miRs by endothelial cells and demonstrated a potential function for platelet miR-320b in the regulation of endothelial expression of intercellular adhesion molecule-1 (ICAM-1). ${ }^{39}$ Laffont et al showed that also the most abundant platelet microRNA, miR-223, could be trans- ferred to the endothelium in this way. ${ }^{40}$ The authors demonstrated regulation of both mRNA and protein expression of two miR-223 targets, F-box/WD repeat-containing protein 7 (FBXW7), and ephrin A1 (EFNA1), in endothelial cells. Pan et al also looked at transfer of miR-223 to endothelial cells and showed that miR-223 promotes endothelial cell apoptosis by targeting the insulin-like growth factor 1 receptor. ${ }^{41} \mathrm{~A}$ more recent study by Li et al looked at transfer of platelet miR-223 upon activation by thrombin to human umbilical vein endothelial cells (HUVECs) and found that miR-223 also downregulated endothelial ICAM-1 via inhibition of p38, cJun N-terminal kinase, and extracellular signal-regulated kinase phosphorylation, and thereby dampens a thrombosis-induced inflammatory response. ${ }^{42}$ Zhang et al demonstrated that antibodies against the $\beta 2$-glycoprotein 1 (B2GP1)/B2GP1 complex induced the release and concurrent endothelial uptake of platelet miR-96 and miR-26a. ${ }^{43}$ In HUVECs, miR-96 and miR-26c targeted P-selectin and the platelet-derived growth factor (PDGF) $\alpha$-receptor and inhibited migration and tube formation (-Table $\mathbf{2}$ ).

Platelet miRs are not only transferred to endothelial cells. Tan et al demonstrated that SMCs could take up miR-223, miR339 , and miR-21 from platelet-derived exosomes in vitro. ${ }^{44}$ Once inside the cell, these miRs targeted PDGF receptor (PDGFR) $\beta$ and inhibited PDGF-induced SMC proliferation. In a very recent study, Yang et al showed that macrophages can also take up platelet MVs. ${ }^{4}$ Instead of being degraded, miRs from these vesicles, in this study demonstrated for miR-4306, a marker for poor prognosis in patients with CAD, directly influenced macrophage functionality. Platelet-derived miR4306 reduced cardiac tissue infiltration by macrophages in a murine myocardial infarction model (- Table 2).

HUVECS and SMCs are commonly used in vitro model systems for the human vasculature. However, to our knowledge, no study has provided direct evidence yet for in vivo transfer of platelet miRs into the vessel wall. There is evidence of in vivo transfer of platelet mRNA from vesicles to circulating monocytes, but it cannot be fully excluded that this is an effect of phagocytosis, rather than active uptake. ${ }^{46}$

Because of their presence in the blood stream, platelets would be relatively easy cellular targets for anti-microRNAbased therapeutics. However, there is a serious drawback to such strategies however, as Flierl et al demonstrated that phosphorothioate backbone modifications, commonly used to stabilize antisense microRNA inhibitors, were potent activators of platelets. ${ }^{47}$

\section{Platelets microRNAs in Antiplatelet Drug Resistance}

As described above, platelets secrete MVs containing miRs upon activation and different platelet agonists lead to different microRNA signatures. As miRs are relatively stable in the circulation, ${ }^{3}$ this renders platelet miRs highly suitable as potential biomarkers. Platelet miRs are increased in the circulation in patients with $C A D,{ }^{48}$ as will be described further below. Many studies have also focused on the potential of platelet miRs as markers for a common problem in 
treatment of patients with CAD, namely antiplatelet drug resistance.

Willeit et al first investigated the response of plateletderived miRs in plasma of healthy volunteers treated with antiplatelet drugs (prasugrel and/or aspirin), ${ }^{49}$ after which they confirmed their findings in 33 patients with symptomatic carotid atherosclerosis. The authors found that particularly miR-223 and miR-191, as well as miR-126 and miR-150, were downregulated during antiplatelet therapy both in patients with carotid atherosclerosis and in healthy controls. This finding was confirmed for miR-126 by De Boer et al in plasma from patients with type 2 diabetes. ${ }^{50}$ Shi et al also looked at levels of miR-223 and miR-96 in CAD patients on antiplatelet therapy, but here the authors also included platelet responsiveness. ${ }^{51}$ They showed that miR-223 levels in platelets were correlated directly with platelet responsiveness to clopidogrel. Platelet miR-223 expression was also associated with high on-treatment platelet reactivity in acute coronary syndrome (ACS) patients. ${ }^{52}$ This correlation between miR-223 and platelet responsiveness was confirmed by several other studies. ${ }^{32,53,54}$ Similarly, several other platelet miRs were reported to correlate with ontreatment platelet reactivity, including miR-126, miR-92a, miR-26a, and miR-365-3p. ${ }^{32,55-57}$ MiR-126 and miR-223 showed strong correlation with platelet function tests in plasma of patients on dual antiplatelet therapy 1 month after ACS. $^{32}$ Furthermore, inhibition of miR-126 leads to reduced platelet aggregation and receptor expression in mice. ${ }^{32}$ Three platelet-related miRs, miR-223, miR-221, and miR21 , were associated with platelet responsiveness on clopidogrel treatment in platelets of carriers of CYP2C $19 * 2$ genotype following ACS and discriminate high and low responders on clopidogrel treatment. ${ }^{54}$ After therapeutic switch from clopidogrel to ticagrelor, expression of miR126 , miR-223, and miR-150 in plasma decreases, whereas the expression of miR-96 increases. ${ }^{58}$ In addition, miR-126 expression was further decreased in plasma from patients with ticagrelor loading as compared with patients without loading dose, suggesting that miR-126 expression decreases with increasing intensity of platelet inhibition. ${ }^{58}$ A very recent study by La Rosa et al described a potential mechanism for antiplatelet drug resistance. ${ }^{59}$ The authors showed that chronic aspirin treatment leads to the downregulation of miR-26b-5p, which the authors demonstrate to target the multidrug resistance protein-4 (MRP4) in platelets. Increased MRP4 levels had previously been linked to reduced platelet responsiveness to aspirin treatment. ${ }^{60}$

\section{Platelet microRNAs in Coronary Artery Disease}

Parallel to bench studies demonstrating the role of miRs in regulation of platelet function and reactivity, the number of studies investigating clinical utility of circulating plateletrelated miRs in CAD has increased. Several platelet-related miRs have been proposed as diagnostic biomarkers for ACS either alone or in combination with other miRs. In one of the first studies in patients with CAD, Fichtlscherer et al have demonstrated reduced expression of miR-126 and miR-199 as well as other vascular- and inflammation-associated miRs as compared with healthy controls. ${ }^{10}$ In one other study, miR-126 could not discriminate patients with CAD from patients without CAD. ${ }^{61}$

Circulating miRs were also proposed as potential diagnostic biomarkers in the setting of AMI, as changes in expression levels of circulating miRs are detectable very early after symptom onset. Levels of miR-126 are reduced, whereas plasma levels of miR-21 and miR-186 peaked within 4 hours after symptom onset, suggesting the potential role of platelet-related miRs in early identification of AMI patients. ${ }^{62-64}$ Furthermore, circulating miR-223 is increased in patients with AMI as compared with angina pectoris and controls. ${ }^{65}$ In addition, a miRs signature consisting of miR223 and 5 other cardiac-enriched miRs showed similar discriminatory power for AMI as high sensitivity cardiac troponin (hs-cTn). ${ }^{65}$ In contrast to these results, De Rosa et al found no difference in miR-223 and miR-126 expression levels between ACS, stable CAD, and controls, whereas Corsten et al detected decreased expression of miR-223 in AMI patients as compared with controls. ${ }^{66}$

Several other platelet-related miRs were also associated with AMI. Patients with AMI had increased expression levels of circulating miR-221, which could discriminate AMI from controls with area under the curve (AUC) of $0.881 .^{67}$ The expression of miR-21 is increased in ACS patients as compared with non-ACS, ${ }^{68-70}$ and miR-21 levels correlated with hs-cTn in AMI patients. ${ }^{71}$

Not only plasma, but also platelet miRs expression profile differs in ST-elevation myocardial infarction (STEMI) compared with non-ST-elevation myocardial infarction (NSTEMI) patients. Ward et al could show that miR-221 and miR-25 is upregulated in platelets of STEMI patients as compared with NSTEMI, whereas the expression of miR-186 and miR-342 was reduced. ${ }^{72}$ Furthermore, STEMI patients have lower platelet expression of miR-126 and miR-21, and higher expression of miR-223 and miR-150, and platelet miR-126 showed inverse correlation with hs-cTn. ${ }^{73}$ In contrast to high platelet expression in AMI, the expression of miR-223 in monocytes is downregulated in AMI, and returns to previous levels after 3 months. ${ }^{74}$ Moreover, platelets alter their miRs expression profile after cardiopulmonary bypass for elective cardiac surgery. Mukai et al could show that overexpression of miR-10b and miR-96 in platelets reduces the GP1b and vesicle-associated membrane protein $8 \mathrm{mRNA}$ and protein expression, possibly altering platelet function after cardiopulmonary bypass. ${ }^{75}$

However, none of the large studies could demonstrate the benefit of assessing circulating miRs in addition to hs-cTn for diagnosis of AMI. Even in the early presenters, it is extremely difficult for new biomarkers to outperform hs-cTn. To date, none of the miRs proposed as marker for early AMI detection could outperform hs-cTn with respect to sensitivity and specificity. However, since increased sensitivity of new hscTn comes at the price of reduced specificity, accurate discrimination of patients with NSTEMI and unstable angina from other causes of chest pain with rapid "rule-in" and 
Table 1 Overview of key platelet microRNAs and their function within platelets

\begin{tabular}{|c|c|c|c|c|}
\hline microRNA & Target & Role in platelets & Study samples & Study \\
\hline miR-223 & $\begin{array}{l}\text { Factor XIII } \\
\text { P2Y12 receptor }\end{array}$ & Platelet aggregation & $\begin{array}{l}\text { Mice; } \\
\text { Human platelets }\end{array}$ & $\begin{array}{l}\text { Elgheznawy et al } \\
\text { Fejes et } \mathrm{al}^{30}\end{array}$ \\
\hline miR-126 & $\begin{array}{l}\text { Disintegrin and } \\
\text { metalloproteinase } \\
\text { domain-containing } \\
\text { protein 9, } \\
\text { Plexin-B2 }\end{array}$ & Platelet reactivity & Human megakaryocytes & $\begin{array}{l}\text { Kaudewitz et al } \\
\text { Garcia et } \mathrm{al}^{31}\end{array}$ \\
\hline miR-21 & $\begin{array}{l}\text { Wiskott-Aldrich } \\
\text { syndrome protein }\end{array}$ & TGF- $\beta 1$ secretion & Mice & Barwari et al ${ }^{37}$ \\
\hline miR-30c & PAI-1 & PAl-1 production & $\begin{array}{l}\text { Mice; } \\
\text { Human }\end{array}$ & Luo et $\mathrm{al}^{29}$ \\
\hline miR-96 & VAMP8 & Platelet reactivity & Human platelets & Kondkar et al ${ }^{33}$ \\
\hline miR-148a & $\begin{array}{l}\text { T cell ubiquitin li- } \\
\text { gand-2 }\end{array}$ & Activation via the Fc receptor for IgG IIA & Mice & Zhou et $\mathrm{al}^{34}$ \\
\hline $\operatorname{miR}-376 c$ & PC-TP & Platelet reactivity & Humans & Edelstein et $\mathrm{al}^{24}$ \\
\hline
\end{tabular}

Abbreviations: IgG, immunoglobulin G; PAI-1, plasminogen activator inhibitor-1; PC-TP, phosphatidylcholine transfer protein; RNA, ribonucleic acid; TGF- $\beta 1$, transforming growth factor- $\beta 1$.

Table 2 Overview of key platelet microRNAs and their function outside platelets

\begin{tabular}{|l|l|l|l|l|}
\hline microRNA & Target protein & Target cells & Study samples & Study \\
\hline miR-320b & ICAM1 & Endothelial cells & HMEC-1 & Gidlöf et al $^{39}$ \\
\hline miR-223 & FBXW7/EFNA1 & Endothelial cells & HUVECs & Laffont et al $^{40}$ \\
\hline miR-223 & ILGFR1 & Endothelial cells & HUVECs & Pan et al $^{41}$ \\
\hline miR-223 & ICAM1 & Endothelial cells & HUVECs & i et al $^{42}$ \\
\hline $\begin{array}{l}\text { miR-96, } \\
\text { miR-26a }\end{array}$ & PDGFRA & Endothelial cells & HUVECs & Zhang et al $^{43}$ \\
\hline $\begin{array}{l}\text { miR-223, } \\
\text { miR-339, }\end{array}$ & PDGFRB & Smooth muscle cells & $\begin{array}{l}\text { Primary murine } \\
\text { aorta SMCs }\end{array}$ & Tan et al \\
\hline \begin{tabular}{l} 
miR-4306 \\
\hline
\end{tabular} & VEGFA & Monocyte-derived macrophages & $\begin{array}{l}\text { Human peripheral blood } \\
\text { mononuclear cells }\end{array}$ & Yang et al \\
\hline
\end{tabular}

Abbreviations: HMEC-1, human microvascular endothelial cell 1; HUVEC, human umbilical vein endothelial cell; ICAM1, intercellular adhesion molecule-1; RNA, ribonucleic acid; SMC, smooth muscle cell; VEGFA, vascular endothelial growth factor A.

"rule-out" remains an unmet clinical need. ${ }^{76-78}$ Furthermore, it is tempting to speculate that a specific miRs signature rather than one standalone microRNA, if at all, might improve specificity and add diagnostic information on top of hs-cTn and clinical risk factors. This is particularly relevant in the aforementioned clinical setting of suspected ACS in whom clinical, laboratory, and electrocardiography (ECG) findings are inconclusive. In a large, multicenter study with 1,155 acute chest pain patients, miR-223 could not discriminate AMI from other causes of chest pain. ${ }^{79}$ In fact, none of the investigated miRs had any incremental diagnostic value when added to hs-cTn. ${ }^{79}$ However, a miRs signature consisting of cardiac and platelet-related miRs, miR-1, miR-21, and miR-499, showed better diagnostic performance than hs-cTn alone, and increased diagnostic accuracy of hs-cTn and clinical risk factors. ${ }^{68}$ This effect was especially pronounced in early presenters and in patients with initially negative troponin. Zeller et al identified differential expression of 8 circulating miRs in patients with unstable angina, and proposed a panel of 3 miRs consisting of miR-132, miR-150, and miR-186 for discrimination of patients with unstable angina from other causes of chest pain with high discriminatory power (AUC: 0.91 ; 95\% confidence interval: $0.84-0.98$ ). The diagnostic accuracy of the 3 miRs signature was superior to combination of hs-cTn, brain natriuretic peptide, C-reactive protein, and cystatin C, respectively. ${ }^{80}$ Thus, platelet-related miRs might have diagnostic potential as part of miRs signature in patients with unstable angina or early presenters with initially negative hs-cTn.

In one of the largest studies to date evaluating prognostic value of circulating miRs in patients with CAD, Karakas et al identified a miRs signature consisting of platelet-related miR-210, together with miR-132 and miR-140 as independent predictors of cardiovascular death after 4 years' followup in a large cohort of over 1,000 patients with documented CAD: 483 ACS patients and 682 patients with stable angina. ${ }^{81}$ 
The predictive power was more pronounced in the subgroup of patients with ACS. A combination of several circulating miRs did not provide additional prognostic information when compared with single miRs. ${ }^{81}$ Moreover, in one other study, platelet-related miR-197 and miR-223 also predicted cardiovascular death in patients with documented CAD. ${ }^{82}$ Similarly to the study of Karakas et al, the prognostic power was more pronounced in the subgroup of patients with previous ACS and there was no benefit of analyzing both miRs compared with single miR-197 or miR-223. ${ }^{81,82}$ In patients with CAD on dual antiplatelet therapy, out of 5 investigated platelet-related miRs, only miR-126 was an independent predictor for major adverse cardiovascular events (MACEs) 1 year after percutaneous coronary intervention. ${ }^{83}$ Interestingly, increased expression of miR-126 and miR-199 in circulating endothelial- and platelet-derived MVs, but not in plasma, was associated with a lower risk for future MACE in patients with stable CAD ${ }^{84}$ Since miRs released from activated platelets can regulate expression of adhesion molecules in endothelial cells, ${ }^{39}$ one could speculate that packaging of functional miRs within circulating MVs might influence intercellular signaling and future clinical outcome. In a prospective case-control study, focusing only on STEMI patients, Jakob et al identified miR-26b, miR-320a, and miR-660 as prognostic biomarkers for MACEs after STEMI. All 3 miRs were superior to hs-cTn in risk stratification of STEMI patients and added prognostic information on top of Global Registry of Acute Coronary Events score and hs$\mathrm{cTn}^{85}$ MiR-26b and miR320a were previously shown to play a role in ventricular remodeling in heart failure, whereas miR-660 may have a prothrombotic effect by increasing production of activated platelets. ${ }^{86-88}$

A signature of 3 platelet-related miRs, namely miR-126, miR-197, and miR-223, has been proposed as prognostic marker for occurrence of AMI within 10 years of follow-up in a population-based study. ${ }^{89}$ Assessment of these 3 plateletrelated miRs improved risk stratification when added to the Framingham Risk Score. ${ }^{89}$ The expression levels of miR-223 and miR-126 are reduced in smokers and diabetic patients, ${ }^{12,90}$ suggesting that most important cardiovascular risk factors alter the expression profile of platelet-related miRs. In another smaller population study in apparently healthy subjects, a combination of let-7g, miR-106a, miR424, miR-144, and miR-660 was associated with increased risk of AMI after 10 years' follow-up, and improved the AUC when added to the Framingham Risk Score. ${ }^{91}$ Thus, circulating, platelet-related miRs as standalone markers or as part of miRs signatures might be used for risk stratification in both primary and secondary prevention.

\section{Current Challenges and Future Perspectives}

Although a growing amount of data suggests a high potential for the use of miRs as diagnostic and prognostic biomarkers in AMI, its routine use in clinical practice remains limited so far. Analysis of circulating miRs is still time consuming, expensive, and depends on availability of specific laboratory resources. Several aspects of sample handling and prepara- tion may influence the results of the experiment. Rapid and precise sample collection as well as adequate sample storage is necessary to obtain high-quality RNA. ${ }^{92}$ Medication use, such as heparin or antiplatelet drugs, may interfere with quantification of miRs or alter their expression. ${ }^{49,50,93,94}$ This is of particular importance in STEMI patients, because application of intravenous heparin or loading with platelet inhibitors before sample collection is clinical routine and may tamper the results. Previous studies have used mostly serum or plasma samples as starting material. Because of the clotting process and platelet activation, the expression of platelet-related miRs and MVs is increased in serum samples. ${ }^{95}$ Thus, ethylenediaminetetraacetic acid plasma samples are most suitable as starting material for subsequent miRs quantification. ${ }^{95}$ Due to its high sensitivity, specificity, and precision, quantitative real-time polymerase chain reaction (PCR) is considered gold standard for assessment of miRs. ${ }^{96}$ Data obtained by quantitative real-time PCR have to be normalized to account for variability during sample preparation. Several data normalization methods have been proposed, such as endogenous or exogenous controls, or $\mathrm{Ct}$ average of all measured miRs. Currently, there is no consensus on the optimal normalization method, and the use of several controls is recommended. ${ }^{95}$ However, the usage of different starting materials, quantification, and normalization methods results in high heterogeneity of the studies and makes the comparison of studies extremely difficult. A consensus on minimal standards regarding sample collection, RNA preparation, quantification, data normalization, and analysis is necessary to bring circulating miRs as biomarkers closer to clinical practice.

Beside technical aspects, there is still much to learn about physiological regulation of circulating miRs. Mechanism of miRs release into and uptake from the circulation are poorly understood so far. Comorbidities, such as arterial hypertension, cancer, diabetes, or impaired renal function, might also alter the expression of miRs and must be accounted for in the statistical analyses. ${ }^{97-99}$ Circulating miRs are also affected by the use of statins or angiotensin converting enzyme inhibitors, both important agents in secondary prevention of CAD. ${ }^{100,101}$ Since miRs are packed and transported with HDL, changes of the cholesterol profile of patients by food intake or use of statins may further alter the expression of circulating miRs. Furthermore, accurate discrimination of type 1 and type 2 AMI is challenging in everyday clinical practice. According to the current universal AMI definition, type 1 and type 2 AMI have different pathophysiological background (coronary plaque rupture vs. imbalance of myocardial oxygen supply and demand). Currently, there are no studies investigating the role of miRs in type 2 AMI and future studies should assess the diagnostic value of circulating miRs in this heterogeneous patient population with high cardiovascular risk.

\section{Conclusion}

Platelets express functional miRs which can in turn regulate platelet function and reactivity, as well as intercellular 
communication. Specific miRs signature might improve diagnostic accuracy in patients with suspected ACS in whom clinical, laboratory, and ECG findings are inconclusive. Furthermore, circulating miRs might also improve risk stratification of patients following ACS by providing valuable prognostic information on top of clinical judgment and cardiovascular risk factors. Finally, platelet-related miRs have remarkable potential as biomarkers for treatment monitoring and therapy response in patients on antiplatelet medication. However, before its implementation into daily clinical routine, a better understanding of the physiology of circulating miRs, as well as improved time- and cost-effective analytical procedures are required.

\section{Conflict of Interest}

None declared.

\section{References}

1 Baek D, Villén J, Shin C, Camargo FD, Gygi SP, Bartel DP. The impact of microRNAs on protein output. Nature 2008;455 (7209):64-71

2 Bartel DP. MicroRNAs: target recognition and regulatory functions. Cell 2009;136(02):215-233

3 Mitchell PS, Parkin RK, Kroh EM, et al. Circulating microRNAs as stable blood-based markers for cancer detection. Proc Natl Acad Sci U S A 2008;105(30):10513-10518

4 Valadi H, Ekström K, Bossios A, Sjöstrand M, Lee JJ, Lötvall JO. Exosome-mediated transfer of mRNAs and microRNAs is a novel mechanism of genetic exchange between cells. Nat Cell Biol 2007;9(06):654-659

5 Hunter MP, Ismail N, Zhang X, et al. Detection of microRNA expression in human peripheral blood microvesicles. PLoS One 2008;3(11):e3694

6 Vickers KC, Palmisano BT, Shoucri BM, Shamburek RD, Remaley AT. MicroRNAs are transported in plasma and delivered to recipient cells by high-density lipoproteins. Nat Cell Biol 2011; 13(04):423-433

7 Turchinovich A, Weiz L, Langheinz A, Burwinkel B. Characterization of extracellular circulating microRNA. Nucleic Acids Res 2011;39(16):7223-7233

8 Wang GK, Zhu JQ, Zhang JT, et al. Circulating microRNA: a novel potential biomarker for early diagnosis of acute myocardial infarction in humans. Eur Heart J 2010;31(06): 659-666

9 D’Alessandra Y, Devanna P, Limana F, et al. Circulating microRNAs are new and sensitive biomarkers of myocardial infarction. Eur Heart J 2010;31(22):2765-2773

10 Fichtlscherer S, De Rosa S, Fox H, et al. Circulating microRNAs in patients with coronary artery disease. Circ Res 2010;107(05): 677-684

11 Tijsen AJ, Creemers EE, Moerland PD, et al. MiR423-5p as a circulating biomarker for heart failure. Circ Res 2010;106(06): 1035-1039

12 Zampetaki A, Kiechl S, Drozdov I, et al. Plasma microRNA profiling reveals loss of endothelial miR-126 and other microRNAs in type 2 diabetes. Circ Res 2010;107(06): 810-817

13 Stojkovic S, Jurisic M, Kopp CW, et al. Circulating microRNAs identify patients at increased risk of in-stent restenosis after peripheral angioplasty with stent implantation. Atherosclerosis 2018;269:197-203

14 Ahlin F, Arfvidsson J, Vargas KG, Stojkovic S, Huber K, Wojta J. MicroRNAs as circulating biomarkers in acute coronary syndromes: a review. Vascul Pharmacol 2016;81:15-21
15 Hohensinner PJ, Kaun C, Ebenbauer B, et al. Reduction of premature aging markers after gastric bypass surgery in morbidly obese patients. Obes Surg 2018;28(09):2804-2810

16 Merkerova M, Belickova M, Bruchova H. Differential expression of microRNAs in hematopoietic cell lineages. Eur J Haematol 2008;81(04):304-310

17 Landry P, Plante I, Ouellet DL, Perron MP, Rousseau G, Provost P. Existence of a microRNA pathway in anucleate platelets. Nat Struct Mol Biol 2009;16(09):961-966

18 Rowley JW, Chappaz S, Corduan A, et al. Dicer1-mediated miRNA processing shapes the mRNA profile and function of murine platelets. Blood 2016;127(14):1743-1751

19 Nagalla S, Shaw C, Kong X, et al. Platelet microRNA-mRNA coexpression profiles correlate with platelet reactivity. Blood 2011;117(19):5189-5197

20 Osman A, Fälker K. Characterization of human platelet microRNA by quantitative PCR coupled with an annotation network for predicted target genes. Platelets 2011;22(06):433-441

21 Stratz C, Nührenberg TG, Binder H, et al. Micro-array profiling exhibits remarkable intra-individual stability of human platelet micro-RNA. Thromb Haemost 2012;107(04):634-641

22 Plé H, Landry P, Benham A, Coarfa C, Gunaratne PH, Provost P. The repertoire and features of human platelet microRNAs. PLoS One 2012;7(12):e50746

23 Simon LM, Edelstein LC, Nagalla S, et al. Human platelet microRNA-mRNA networks associated with age and gender revealed by integrated plateletomics. Blood 2014;123(16):e37-e45

24 Edelstein LC, Simon LM, Montoya RT, et al. Racial differences in human platelet PAR4 reactivity reflect expression of PCTP and miR-376c. Nat Med 2013;19(12):1609-1616

25 Nassa G, Giurato G, Cimmino G, et al. Splicing of platelet resident pre-mRNAs upon activation by physiological stimuli results in functionally relevant proteome modifications. Sci Rep 2018;8 (01):498

26 Elgheznawy A, Shi L, Hu J, et al. Dicer cleavage by calpain determines platelet microRNA levels and function in diabetes. Circ Res 2015;117(02):157-165

27 Leierseder S, Petzold T, Zhang L, Loyer X, Massberg S, Engelhardt $\mathrm{S}$. MiR-223 is dispensable for platelet production and function in mice. Thromb Haemost 2013;110(06):1207-1214

28 Welten SM, Goossens EA, Quax PH, Nossent AY. The multifactorial nature of microRNAs in vascular remodelling. Cardiovasc Res 2016;110(01):6-22

29 Luo M, Li R, Ren M, et al. Hyperglycaemia-induced reciprocal changes in miR-30c and PAI-1 expression in platelets. Sci Rep 2016;6:36687

30 Fejes Z, Póliska S, Czimmerer Z, et al. Hyperglycaemia suppresses microRNA expression in platelets to increase P2RY12 and SELP levels in type 2 diabetes mellitus. Thromb Haemost 2017;117 (03):529-542

31 Garcia A, Dunoyer-Geindre S, Zapilko V, Nolli S, Reny JL, Fontana P. Functional validation of microRNA-126-3p as a platelet reactivity regulator using human haematopoietic stem cells. Thromb Haemost 2019;119(02):254-263

32 Kaudewitz D, Skroblin P, Bender LH, et al. Association of microRNAs and YRNAs with platelet function. Circ Res 2016;118(03): 420-432

33 Kondkar AA, Bray MS, Leal SM, et al. VAMP8/endobrevin is overexpressed in hyperreactive human platelets: suggested role for platelet microRNA. J Thromb Haemost 2010;8(02): 369-378

34 Zhou Y, Abraham S, Andre P, et al. Anti-miR-148a regulates platelet FcrRIIA signaling and decreases thrombosis in vivo in mice. Blood 2015;126(26):2871-2881

35 Miao X, Rahman MF, Jiang L, et al. Thrombin-reduced miR-27b attenuates platelet angiogenic activities in vitro via enhancing platelet synthesis of anti-angiogenic thrombospondin-1. J Thromb Haemost 2018;16(04):791-801 
36 Thum T, Gross C, Fiedler J, et al. MicroRNA-21 contributes to myocardial disease by stimulating MAP kinase signalling in fibroblasts. Nature 2008;456(7224):980-984

37 Barwari T, Eminaga S, Mayr U, et al. Inhibition of profibrotic microRNA-21 affects platelets and their releasate. JCI Insight 2018;3(21):3

38 Ambrose AR, Alsahli MA, Kurmani SA, Goodall AH. Comparison of the release of microRNAs and extracellular vesicles from platelets in response to different agonists. Platelets 2018;29 (05):446-454

39 Gidlöf O, van der Brug M, Ohman J, et al. Platelets activated during myocardial infarction release functional miRNA, which can be taken up by endothelial cells and regulate ICAM1 expression. Blood 2013;121(19):3908-3917

40 Laffont B, Corduan A, Plé $\mathrm{H}$, et al. Activated platelets can deliver mRNA regulatory Ago2 $\bullet$ microRNA complexes to endothelial cells via microparticles. Blood 2013;122(02):253-261

41 Pan Y, Liang $\mathrm{H}$, Liu $\mathrm{H}$, et al. Platelet-secreted microRNA-223 promotes endothelial cell apoptosis induced by advanced glycation end products via targeting the insulin-like growth factor 1 receptor. J Immunol 2014;192(01):437-446

42 Li J, Tan M, Xiang Q, Zhou Z, Yan H. Thrombin-activated plateletderived exosomes regulate endothelial cell expression of ICAM-1 via microRNA-223 during the thrombosis-inflammation response. Thromb Res 2017;154:96-105

43 Zhang Y, Zhang W, Zha C, Liu Y. Platelets activated by the anti$\beta 2 \mathrm{GPI} / \beta 2 \mathrm{GPI}$ complex release microRNAs to inhibit migration and tube formation of human umbilical vein endothelial cells. Cell Mol Biol Lett 2018;23:24

44 Tan M, Yan HB, Li JN, et al. Thrombin stimulated platelet-derived exosomes inhibit platelet-derived growth factor receptor-beta expression in vascular smooth muscle cells. Cell Physiol Biochem 2016;38(06):2348-2365

45 Yang Y, Luo H, Liu S, et al. Platelet microparticles-containing miR4306 inhibits human monocyte-derived macrophages migration through VEGFA/ERK1/2/NF-KB signaling pathways. Clin Exp Hypertens 2019;41(05):481-491

46 Risitano A, Beaulieu LM, Vitseva O, Freedman JE. Platelets and platelet-like particles mediate intercellular RNA transfer. Blood 2012;119(26):6288-6295

47 Flierl U, Nero TL, Lim B, et al. Phosphorothioate backbone modifications of nucleotide-based drugs are potent platelet activators. J Exp Med 2015;212(02):129-137

48 Sondermeijer BM, Bakker A, Halliani A, et al. Platelets in patients with premature coronary artery disease exhibit upregulation of miRNA340* and miRNA624*. PLoS One 2011;6(10):e25946

49 Willeit P, Zampetaki A, Dudek K, et al. Circulating microRNAs as novel biomarkers for platelet activation. Circ Res 2013;112(04): 595-600

50 de Boer HC, van Solingen C, Prins J, et al. Aspirin treatment hampers the use of plasma microRNA-126 as a biomarker for the progression of vascular disease. Eur Heart J 2013;34(44): 3451-3457

51 Shi R, Ge L, Zhou X, et al. Decreased platelet miR-223 expression is associated with high on-clopidogrel platelet reactivity. Thromb Res 2013;131(06):508-513

52 Zhang YY, Zhou X, Ji WJ, et al. Decreased circulating microRNA223 level predicts high on-treatment platelet reactivity in patients with troponin-negative non-ST elevation acute coronary syndrome. J Thromb Thrombolysis 2014;38(01):65-72

53 Chyrchel B, Totoń-Żurańska J, Kruszelnicka O, et al. Association of plasma miR-223 and platelet reactivity in patients with coronary artery disease on dual antiplatelet therapy: a preliminary report. Platelets 2015;26(06):593-597

54 Peng L, Liu J, Qin L, et al. Interaction between platelet-derived microRNAs and CYP2C19*2 genotype on clopidogrel antiplatelet responsiveness in patients with ACS. Thromb Res 2017;157:97-102
55 Binderup HG, Houlind K, Madsen JS, Brasen CL. Aspirin resistance may be identified by miR-92a in plasma combined with platelet distribution width. Clin Biochem 2016;49(15):1167-1172

56 Chen S, Qi X, Chen H, et al. Expression of miRNA-26a in platelets is associated with clopidogrel resistance following coronary stenting. Exp Ther Med 2016;12(01):518-524

57 Chen YC, Lin FY, Lin YW, et al. Platelet microRNA 365-3p expression correlates with high on-treatment platelet reactivity in coronary artery disease patients. Cardiovasc Drugs Ther 2019; 33(02):129-137

58 Carino A, De Rosa S, Sorrentino S, et al. Modulation of circulating microRNAs levels during the switch from clopidogrel to ticagrelor. BioMed Res Int 2016;2016:3968206

59 La Rosa G, Biasucci LM, Mandolini C, et al. Platelet miRNA-26b down-regulates multidrug resistance protein 4 in patients on chronic aspirin treatment. J Cardiovasc Med (Hagerstown) 2018; 19(10):611-613

60 Massimi I, Lotti LV, Temperilli F, et al. Enhanced platelet MRP4 expression and correlation with platelet function in patients under chronic aspirin treatment. Thromb Haemost 2016;116 (06): $1100-1110$

61 Sun X, Zhang M, Sanagawa A, et al. Circulating microRNA-126 in patients with coronary artery disease: correlation with LDL cholesterol. Thromb J 2012;10(01):16

62 Long G, Wang F, Duan Q, et al. Human circulating microRNA-1 and microRNA-126 as potential novel indicators for acute myocardial infarction. Int J Biol Sci 2012;8(06):811-818

63 Wang KJ, Zhao X, Liu YZ, et al. Circulating MiR-19b-3p, MiR-134$5 p$ and MiR-186-5p are promising novel biomarkers for early diagnosis of acute myocardial infarction. Cell Physiol Biochem 2016;38(03):1015-1029

64 Zile MR, Mehurg SM, Arroyo JE, Stroud RE, DeSantis SM, Spinale FG. Relationship between the temporal profile of plasma microRNA and left ventricular remodeling in patients after myocardial infarction. Circ Cardiovasc Genet 2011;4(06):614-619

65 Li C, Fang Z, Jiang T, et al. Serum microRNAs profile from genomewide serves as a fingerprint for diagnosis of acute myocardial infarction and angina pectoris. BMC Med Genomics 2013;6:16

66 Corsten MF, Dennert R, Jochems S, et al. Circulating microRNA208b and microRNA-499 reflect myocardial damage in cardiovascular disease. Circ Cardiovasc Genet 2010;3(06):499-506

67 Coskunpinar E, Cakmak HA, Kalkan AK, Tiryakioglu NO, Erturk M, Ongen Z. Circulating miR-221-3p as a novel marker for early prediction of acute myocardial infarction. Gene 2016;591(01): 90-96

68 Oerlemans MI, Mosterd A, Dekker MS, et al. Early assessment of acute coronary syndromes in the emergency department: the potential diagnostic value of circulating microRNAs. EMBO Mol Med 2012;4(11):1176-1185

69 Darabi F, Aghaei M, Movahedian A, Pourmoghadas A, Sarrafzadegan N. The role of serum levels of microRNA-21 and matrix metalloproteinase- 9 in patients with acute coronary syndrome. Mol Cell Biochem 2016;422(1-2):51-60

70 Olivieri F, Antonicelli R, Lorenzi M, et al. Diagnostic potential of circulating miR-499-5p in elderly patients with acute non STelevation myocardial infarction. Int J Cardiol 2013;167(02): 531-536

71 Wang F, Long G, Zhao C, et al. Atherosclerosis-related circulating miRNAs as novel and sensitive predictors for acute myocardial infarction. PLoS One 2014;9(09):e105734

72 Ward JA, Esa N, Pidikiti R, et al. Circulating cell and plasma microRNA profiles differ between non-ST-segment and ST-segment-elevation myocardial infarction. Fam Med Med Sci Res 2013;2(02):108

73 Li S, Guo LZ, Kim MH, Han JY, Serebruany V. Platelet microRNA for predicting acute myocardial infarction. J Thromb Thrombolysis $2017 ; 44(04): 556-564$ 
74 Parahuleva MS, Euler G, Mardini A, et al. Identification of microRNAs as potential cellular monocytic biomarkers in the early phase of myocardial infarction: a pilot study. Sci Rep 2017;7(01):15974

75 Mukai N, Nakayama Y, Ishi S, et al. Changes in microRNA expression level of circulating platelets contribute to platelet defect after cardiopulmonary bypass. Crit Care Med 2018;46(08):e761-e767

76 Mair J, Lindahl B, Hammarsten O, et al. How is cardiac troponin released from injured myocardium? Eur Heart J Acute Cardiovasc Care 2018;7(06):553-560

77 Mair J, Lindahl B, Müller C, et al. What to do when you question cardiac troponin values. Eur Heart J Acute Cardiovasc Care 2018; 7(06):577-586

78 Vafaie M, Slagman A, Möckel M, et al. Prognostic value of undetectable hs Troponin $\mathrm{T}$ in suspected acute coronary syndrome. Am J Med 2016;129(03):274-282

79 Devaux Y, Mueller M, Haaf P, et al. Diagnostic and prognostic value of circulating microRNAs in patients with acute chest pain. J Intern Med 2015;277(02):260-271

80 Zeller T, Keller T, Ojeda F, et al. Assessment of microRNAs in patients with unstable angina pectoris. Eur Heart J 2014;35(31): 2106-2114

81 Karakas M, Schulte C, Appelbaum S, et al. Circulating microRNAs strongly predict cardiovascular death in patients with coronary artery disease-results from the large AtheroGene study. Eur Heart J 2017;38(07):516-523

82 Schulte C, Molz S, Appelbaum S, et al. miRNA-197 and miRNA223 predict cardiovascular death in a cohort of patients with symptomatic coronary artery disease. PLoS One 2015;10(12): e0145930

83 Yu XY, Chen JY, Zheng ZW, et al. Plasma miR-126 as a potential marker predicting major adverse cardiac events in dual antiplatelet-treated patients after percutaneous coronary intervention. EuroIntervention 2013;9(05):546-554

84 Jansen F, Yang X, Proebsting S, et al. MicroRNA expression in circulating microvesicles predicts cardiovascular events in patients with coronary artery disease. J Am Heart Assoc 2014; 3(06):e001249

85 Jakob P, Kacprowski T, Briand-Schumacher S, et al. Profiling and validation of circulating microRNAs for cardiovascular events in patients presenting with ST-segment elevation myocardial infarction. Eur Heart J 2017;38(07):511-515

86 Han M, Yang Z, Sayed D, et al. GATA4 expression is primarily regulated via a miR-26b-dependent post-transcriptional mechanism during cardiac hypertrophy. Cardiovasc Res 2012;93(04):645-654

87 Emmrich S, Henke K, Hegermann J, Ochs M, Reinhardt D, Klusmann JH. miRNAs can increase the efficiency of ex vivo platelet generation. Ann Hematol 2012;91(11):1673-1684
88 Ren XP, Wu J, Wang X, et al. MicroRNA-320 is involved in the regulation of cardiac ischemia/reperfusion injury by targeting heat-shock protein 20. Circulation 2009;119(17): 2357-2366

89 Zampetaki A, Willeit P, Tilling L, et al. Prospective study on circulating MicroRNAs and risk of myocardial infarction. J Am Coll Cardiol 2012;60(04):290-299

90 Badrnya S, Baumgartner R, Assinger A. Smoking alters circulating plasma microvesicle pattern and microRNA signatures. Thromb Haemost 2014;112(01):128-136

91 Bye A, Røsjø H, Nauman J, et al. Circulating microRNAs predict future fatal myocardial infarction in healthy individuals - The HUNT study. J Mol Cell Cardiol 2016;97:162-168

92 Häntzsch M, Tolios A, Beutner F, et al. Comparison of whole blood RNA preservation tubes and novel generation RNA extraction kits for analysis of mRNA and MiRNA profiles. PLoS One 2014;9 (12):e113298

93 Boeckel JN, Thomé CE, Leistner D, Zeiher AM, Fichtlscherer S, Dimmeler S. Heparin selectively affects the quantification of microRNAs in human blood samples. Clin Chem 2013;59(07): $1125-1127$

94 Kaudewitz D, Lee R, Willeit P, et al. Impact of intravenous heparin on quantification of circulating microRNAs in patients with coronary artery disease. Thromb Haemost 2013;110(03):609-615

95 Zampetaki A, Mayr M. Analytical challenges and technical limitations in assessing circulating miRNAs. Thromb Haemost 2012; 108(04):592-598

96 Ach RA, Wang H, Curry B. Measuring microRNAs: comparisons of microarray and quantitative PCR measurements, and of different total RNA prep methods. BMC Biotechnol 2008;8:69

97 Gidlöf O, Andersson P, van der Pals J, Götberg M, Erlinge D. Cardiospecific microRNA plasma levels correlate with troponin and cardiac function in patients with ST elevation myocardial infarction, are selectively dependent on renal elimination, and can be detected in urine samples. Cardiology 2011;118(04):217-226

98 Garzon R, Calin GA, Croce CM. MicroRNAs in cancer. Annu Rev Med 2009;60:167-179

99 Willeit P, Skroblin P, Moschen AR, et al. Circulating microRNA-122 is associated with the risk of new-onset metabolic syndrome and type 2 diabetes. Diabetes 2017;66(02): 347-357

100 Li J, Chen H, Ren J, et al. Effects of statin on circulating microRNAome and predicted function regulatory network in patients with unstable angina. BMC Med Genomics 2015;8:12

101 Weber M, Baker MB, Patel RS, Quyyumi AA, Bao G, Searles CD. MicroRNA expression profile in CAD patients and the impact of ACEI/ARB. Cardiol Res Pract 2011;2011:532915 